\title{
IMPACT DU MOMENT DE LA JOURNÉE ET DU RYTHME DE VEILLE- SOMMEIL SUR LES PERFORMANCES COGNITIVES
}

Christina Schmidt, Fabienne Collette

John Libbey Eurotext | « Revue de neuropsychologie»

2016/3 Volume 8 | pages 173 à 181

ISSN 2101-6739

Article disponible en ligne à l'adresse :

https://www.cairn.info/revue-de-neuropsychologie-2016-3-page-173.htm

\section{Pour citer cet article :}

Christina Schmidt, Fabienne Collette Impact du moment de la journée et du rythme de veille-sommeil sur les performances cognitives », Revue de neuropsychologie 2016/3 (Volume 8), p. 173-181.

DOI 10.1684/nrp.2016.0391

Distribution électronique Cairn.info pour John Libbey Eurotext.

(C) John Libbey Eurotext. Tous droits réservés pour tous pays.

La reproduction ou représentation de cet article, notamment par photocopie, n'est autorisée que dans les limites des conditions générales d'utilisation du site ou, le cas échéant, des conditions générales de la licence souscrite par votre établissement. Toute autre reproduction ou représentation, en tout ou partie, sous quelque forme et de quelque manière que ce soit, est interdite sauf accord préalable et écrit de l'éditeur, en dehors des cas prévus par la législation en vigueur en France. Il est précisé que son stockage dans une base de données est également interdit. 
Article de synthèse

Rev Neuropsychol

$2016 ; 8(3): 173-81$

\section{Impact du moment de la journée et du rythme de veille-sommeil sur les performances cognitives}

\section{Impact of time-of-day and sleep-wake cycles on cognitive performance}

Christina Schmidt ${ }^{1,2}$,

Fabienne Collette ${ }^{1,2}$

${ }^{1} \mathrm{GIGA}-\mathrm{CRC}$ In vivo imaging

Université de Liège, Quartier Agora,

Allée du 6-Août $n^{0}$ 8, 4000 Liège, Belgique

${ }^{2}$ Unité de recherche psychologie et

neuroscience cognitives (PsyNCog),

Université de Liège,

Place des Orateurs 1, 4000 Liège, Belgique

<f.collette@ulg.ac.be>

Pour citer cet article : Schmidt C, Collette F. Impact du moment de la journée et du rythme de veille-sommeil sur les performances cognitives. Rev Neuropsychol 2016; 8 (3) : 173-81 doi:10.1684/nrp.2016.0391

\section{Résumé}

Un nombre croissant de données scientifiques suggère que nos performances cognitives peuvent présenter des fluctuations journalières importantes, dépendant des habitudes de veille-sommeil propres à chacun. Ainsi, on peut distinguer schématiquement entre personnes du «matin » et personnes du «soir ». Celles-ci diffèrent notamment dans leurs horaires préférentiels de veille et de sommeil et également dans le moment de la journée auquel leurs capacités cognitives seront les plus efficaces. Malgré l'importance attribuée actuellement à l'hygiène des rythmes veillesommeil, nos contraintes professionnelles et sociales nous imposent fréquemment d'être actif et de nous reposer à contretemps de nos besoins physiologiques. Cette revue essaie de mettre en évidence l'importance des modulations journalières de nos capacités cognitives, les corrélats cérébraux qui les sous-tendent, ainsi que l'existence de différences interindividuelles dans nos habitudes de veille-sommeil. Finalement, les effets de la lumière sur notre comportement de sommeil et sur la qualité de notre éveil seront brièvement décrits, sachant que celle-ci agit comme le facteur principal qui synchronise le temps de sommeil sur l'alternance cyclique du jour et de la nuit.

Mots clés : chronobiologie • rythme circadien • sommeil • cognition • imagerie par résonance magnétique

Abstract
Neurobehavioral performance presents fluctuations over
the 24-hour light-dark cycle, depending on the sleep-wake
habits of an individual. These modulations are contingent upon the chronotype, which
reflects interindividual differences in circadian preference, and particularly upon the syn-
chronicity between the individuals' peak periods of circadian arousal and the time of
the day at which testing occurs. Despite the importance currently attributed to sleep-
wake hygiene, our socio-professional timing constraints frequently impose to be active
and sleep against our physiological needs. This review highlights the importance of diur-
nal modulations in cognitive performance, its cerebral underpinnings and the presence of
interindividual differences. Finally, the effects of light on the sleep-wake cycle and associa-
ted neurobehavioral performance will be briefly described. Light represents the principal
factor synchronizing the sleep-wake rhythm to the light-dark cycle and its beneficial effects
for alertness and neurobehavioral performance have been increasingly investigated in the
past decades.

Key words: chronobiology $\cdot$ circadian rhythms $\cdot$ sleep $\cdot$ cognition $\cdot$ magnetic resonance imaging
Correspondance :

F. Collette 


\section{Introduction}

Chez l'homme, l'éveil et le sommeil se produisent de façon cyclique sur une période de 24 heures. L'homme est une espèce diurne, étant éveillé durant la journée solaire et dormant la nuit, pendant la phase d'obscurité. La quantité, la qualité et l'horaire de ce cycle de veille-sommeil sont déterminés par l'interaction entre au moins deux processus étroitement imbriqués [1] (figure 1). Le processus circadien (voir aussi encadré 1) représente une horloge interne de propension à l'éveil ou au sommeil. Le processus homéostatique quant à lui reflète une accumulation progressive de pression de sommeil, en fonction du nombre d'heures passées à l'éveil. Cette pression de sommeil peut se dissiper uniquement lors d'une période de sommeil. Si un individu vit en synchronie avec sa rythmicité circadienne et n'est pas exposé à une privation de sommeil, des niveaux de vigilance et des performances cognitives relativement stables sont maintenus sur une période d'éveil classique de 16 heures. Il est à noter cependant qu'il existe d'importantes différences interindividuelles dans la façon dont nous réagissons à une privation occasionnelle totale ou partielle de sommeil, ou encore en effectuant un travail de nuit ou posté, provoquant un déséquilibre entre processus homéostatiques et circadiens. D'un individu à l'autre, les réactions peuvent varier $d^{\prime}$ une apparente résistance cognitive jusqu'à une détérioration cognitive majeure [2].

Dans cette revue, nous décrirons l'impact du moment de la journée et de nos cycles de veille-sommeil sur la cognition et ses corrélats cérébraux. Nous aborderons également la question des différences interindividuelles dans la capacité à faire face à un effort cognitif réalisé à contretemps

\section{Encadré 1}

La chronobiologie consiste en l'étude de l'organisation temporelle des organismes. Un organisme vivant n'est pas statique, mais constamment exposé à des changements dynamiques provenant de l'environnement ainsi que de changements émanant de son propre corps. Si ces changements internes se répètent systématiquement de façon périodique, on peut parler de rythmes biologiques. Le rythme circadien présente une période d'environ 24 heures (circa: environ; dies: jour) et est synchronisé sur le cycle de 24 heures régnant sur terre par une série de "donneurs de temps», notamment la lumière. Celle-ci adapte la période du rythme circadien sur exactement 24 heures, permettant une synchronisation entre rythmes endogènes et exogènes. Un rythme est caractérisé par son amplitude, sa période, ainsi que par sa phase. L'amplitude consiste en la différence entre la valeur maximale (ou minimale) et la valeur moyenne du paramètre rythmique étudié. La période peut être définie comme le temps après lequel une phase définie de l'oscillation se reproduit. La phase correspond à l'état d'une oscillation (e.g., son maximum ou minimum) à un point spécifique par rapport à un repère de temps extérieur (e.g., moment de la journée). Les marqueurs classiques pour étudier les rythmes circadiens chez l'homme représentent la modulation de la température corporelle ou encore de la sécrétion endogène de l'hormone mélatonine.

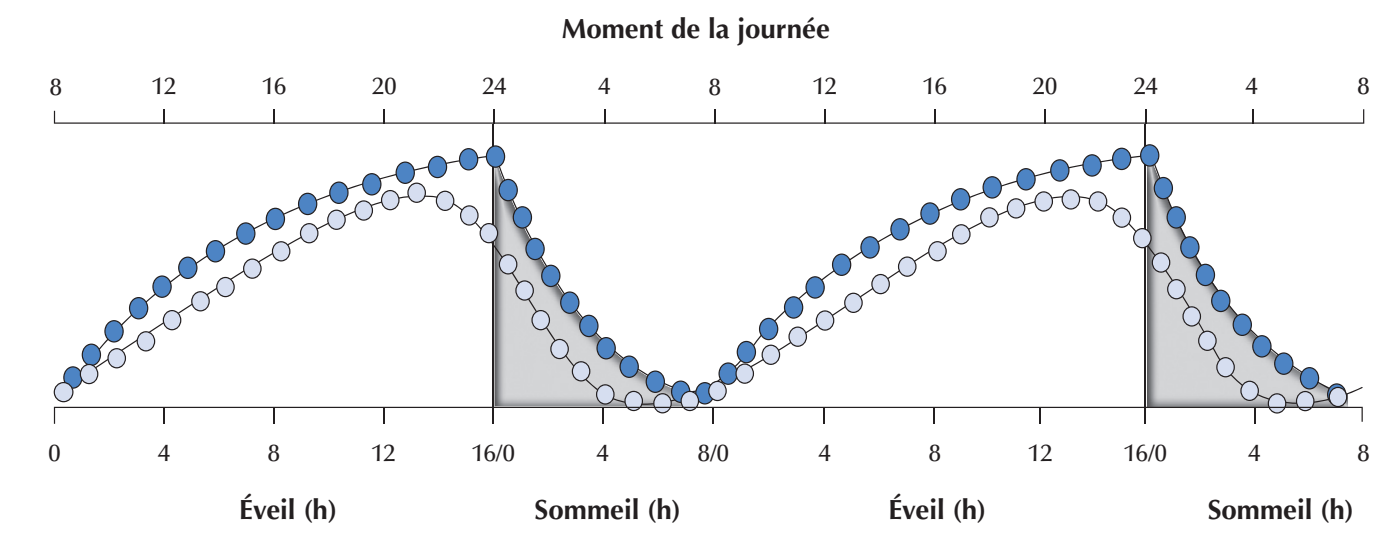

Figure 1. Modèle à deux processus de la régulation du cycle de veille-sommeil (modifié de [1]). Le processus circadien C (gris) oscille avec une phase d'environ 24 heures. Sa propension à l'éveil est maximale en fin de journée, après 12-14 heures d'éveil et minimale en fin de nuit, environ 1-2 heures avant le réveil. Le processus homéostatique de pression de sommeil (bleu) quant à lui augmente continuellement avec le nombre d'heures passés à l'éveil et dissipe de façon exponentielle pendant le sommeil, surtout durant le sommeil lent profond. L'interaction entre ces deux processus détermine la durée et la qualité du sommeil ainsi que de l'éveil. 


\section{Article de synthèse}

de notre horloge biologique interne. Finalement, certains facteurs ayant une influence directe sur notre cycle de veille-sommeil seront détaillés, notamment l'exposition à la lumière. Cette dernière représente, en effet, le principal facteur synchronisant le temps de sommeil sur l'alternance cyclique du jour et de la nuit. À travers son potentiel d'action sur le système circadien, la lumière est actuellement appliquée en tant qu'outil thérapeutique contre une série de troubles liés à une perturbation du cycle de veille-sommeil, et représente également un traitement de premier choix contre la dépression saisonnière. Ces aspects seront brièvement décrits dans la dernière section.

\section{Introduction à la notion de chronobiologie}

Chacun d'entre nous a probablement déjà éprouvé une fatigue irrésistible. La probabilité de ressentir ce genre de creux varie fortement en fonction du moment de la journée. Ainsi, on sait que la survenue de décrochages attentionnels augmente rapidement lorsque la période d'éveil s'étend durant la nuit biologique. D'autres études indiquent également (même si de façon moins consistante) la présence $d^{\prime}$ 'une baisse de performance en début d'après-midi, apparaissant même en absence de repas de midi trop copieux. Au-delà de ces effets bien connus, nous différons également tous dans nos habitudes de veille-sommeil. Une grande variabilité existe, par exemple, dans les horaires préférés de veille et de sommeil, mais aussi dans la manière dont on gère une absence (privation) de sommeil. Ces différents effets se trouvent sous l'influence de rythmes biologiques, des processus investigués par un domaine scientifique spécifique : la chronobiologie (voir encadré 1). Divers rythmes biologiques ont été détectés (rythmes circadiens, circannuels, circalunaires, circatidaux), le plus étudié étant le rythme dit «circadien». Celui-ci possède une période d'environ 24 heures (circa: environ; dies : journée), signifiant qu'il présente un maximum et un minimum se répétant toutes les 24 heures. Le rythme circadien est adapté ou calibré à l'alternance jour/nuit régnant sur terre étant donné la rotation de celle-ci autour de son axe toutes les 24 heures. En effet, on assume que le cycle solaire a provoqué l'évolution de rythmes circadiens chez la majorité des organismes - de la bactérie jusqu'à l'homme - et à tous les niveaux - du gène jusqu'au comportement.

Ces rythmes dits circadiens sont endogènes, $c^{\prime}$ est-à-dire générés par le corps et non provoqués par des influences externes. Ceci fut initialement décrit par de Mairan en 1729 qui a observé que les feuilles d'une plante (Phaseolus coccineus) s'ouvrent et se ferment selon qu'il fait jour ou nuit. Dans un premier temps, il en a conclu que la plante réagissait à la lumière et à l'obscurité. Toutefois, lorsque la plante se trouvait dans des conditions d'obscurité complète, ses feuilles continuaient à se fermer et à s'ouvrir selon un rythme d'environ 24 heures, prouvant sa nature endogène. Le caractère endogène des rythmes circadiens a également été observé chez l'homme pour la première fois en 1938 par Kleitman et Richardson.

Cependant, comme déjà mentionné, le rythme circadien ne présente pas une période d'exactement 24 heures, mais celle-ci est en général légèrement plus longue (en moyenne environ 24,2 heures [3]). Ceci veut dire que si nos habitudes de veille-sommeil étaient uniquement dictées par la rythmicité circadienne endogène, on se coucherait et lèverait chaque jour 0,2 heures plus tard, ce qui provoquerait une désynchronisation entre les conditions extérieures de lumière-obscurité et notre rythme de veille-sommeil. Toutefois, notre rythmicité circadienne est calibrée sur une période exacte de 24 heures à l'aide $d^{\prime}$ « indicateurs temporels» ou Zeitgeber, permettant de synchroniser notre horloge endogène avec le temps externe (voir aussi encadré 1). Le Zeitgeber le plus important représente la lumière environnante. L'information sur la quantité et la qualité de l'exposition à la lumière traverse la rétine pour atteindre l'hypothalamus antérieur (et plus spécifiquement les noyaux suprachiasmatiques, via la voie rétino-hypothalamique [4]). La lumière est ainsi absorbée par des photorécepteurs de l'œil, notamment ceux contenant la mélanopsine [5], puis parvient directement au noyau suprachiasmatique, considéré comme le chef d'orchestre de la rythmicité circadienne. À travers cette voie, la lumière permet de synchroniser notre horloge biologique interne avec les conditions jour-nuit régnant sur terre.

En résumé, les rythmes circadiens sont endogènes, signifiant qu'ils persistent en absence de facteurs externes de l'environnement. Sous ces conditions, leur période dévie légèrement de 24 heures. La présence de Zeitgebers (notamment la lumière du jour) permet leur synchronisation avec le rythme de 24 heures caractérisant la rotation de la terre et l'alternance jour/nuit. Sous des conditions parfaitement synchronisées, l'individu parvient à garder un niveau de vigilance et des performances cognitives relativement stables, même si des différences interindividuelles ont été rapportées. Une désynchronisation entre nos habitudes de vie et nos besoins physiologiques mène à une détérioration de nos états de vigilance et à une perturbation de nos performances cognitives. À côté de la privation de sommeil ou du travail de nuit, un autre exemple de désynchronisation représente le phénomène du jetlag, décrivant les symptômes résultant de l'adaptation de l'organisme à un nouvel horaire, suite à un vol dépassant plusieurs fuseaux horaires par exemple. Dans ce cas, les heures de repas, I'activité et l'endormissement sont décalés pour un individu donné par rapport à ses habitudes. En fonction du décalage, il faut un nombre de jours plus ou moins important pour réajuster ses habitudes de sommeil au nouvel environnement temporel. L'insomnie, le réveil précoce, des troubles de I'humeur, mais aussi de la somnolence journalière associée à des troubles de la vigilance et des facultés d'apprentissage peuvent en résulter.

Dans la section suivante, nous allons préciser plus en détail l'impact du moment de la journée et de nos habitudes de veille-sommeil sur le fonctionnement cognitif. 


\section{Rythmicité circadienne, habitudes de veille-sommeil et cognition}

Les rythmes circadiens s'avèrent d'une nécessité primordiale, puisqu'ils permettent à l'organisme de différencier entre jour et nuit, d'organiser le fonctionnement de toute une série d'organes au cours du temps, mais aussi de contrôler nos performances cognitives.

La qualité de notre sommeil dépend fortement du moment de la journée où il survient. Initier le sommeil en journée va très probablement mener à un sommeil léger et fragmenté, parce que ce moment de la journée se caractérise par un signal circadien promouvant l'éveil et non le sommeil [6]. Le signal circadien d'éveil est le plus prononcé en début de soirée, environ 12-14 heures après le réveil sous des conditions de veille-sommeil classiques, alors que le système circadien favorise le sommeil d'une façon la plus importante en fin de la nuit biologique, environ 1-2 heures avant le réveil (entre 4-6 heures du matin classiquement). La quantité et la qualité de notre sommeil dépendent donc de la phase circadienne, en d'autres mots, du moment de la journée auquel on essaie de l'initier.

Cependant, I'horloge biologique ne détermine pas à elle seule notre niveau de somnolence et notre qualité de veille et de sommeil au cours des 24 heures. Le nombre d'heures passées à l'éveil est un autre facteur déterminant. Plus on reste éveillé, plus une pression de sommeil s'accumule qui ne peut être dissipée que par le sommeil lui-même (figure 1).

Une perturbation dans l'équilibre des processus circadiens et de pression de sommeil peut mener à des troubles du sommeil, de la somnolence et une baisse de la vigilance. Ainsi, les conséquences d'une privation de sommeil s'avèrent les plus néfastes en fin de nuit biologique, dans les alentours de 3 à 6 heures du matin. C'est à ce moment que se sont produits de nombreux accidents tragiques provenant d'une erreur humaine, comme par exemple I'accident nucléaire de Three Mile Island (1979, États Unis), I'accident chimique de Bhopal (1984, Inde) ou encore I'accident nucléaire de Tchernobyl (1986, URSS). Ces accidents reflètent relativement bien le fait que le travail de nuit est «contre-nature » et est responsable, directement ou indirectement, de nombreux accidents et maladies. Dans la même perspective, une étude de Dawson et Reid [7] suggère que la fatigue, tel que provoquée par un sommeil insuffisant ou à une période de sommeil survenant à un moment circadien inapproprié (i.e., en journée), peut provoquer une atteinte des capacités attentionnelles plus importante que la limite du taux d'alcool permis dans le sang pour la conduite automobile (les effets d'une nuit sans sommeil sur les performances attentionnelles seraient en moyenne équivalent à $1 \mathrm{~g} / \mathrm{L}$ d'alcool éthylique dans le sang).

Le rythme circadien affecte donc non seulement la qualité de sommeil, mais aussi notre niveau de fatigue et nos performances durant l'éveil. Déjà en 1885, Ebbinghaus a détecté que la qualité d'apprentissage de syllabes dépendait du moment de la journée. Les premières études à ce sujet se sont focalisées sur l'identification du moment de la journée auquel les capacités d'apprentissages étaient les plus favorables, et ce dans le but d'optimiser les horaires scolaires [8]. Plus tard, Nathaniel Kleitmann, un pionnier dans le domaine de la chronobiologie, a suggéré un parallélisme entre la rythmicité circadienne de la température corporelle et les fluctuations journalières de la performance à des tâches répétitives simples (e.g., substitution de codes, triage de cartes, dessin en miroir [9]). Par la suite, toute une série d'études ont pu mettre en évidence une telle relation temporelle entre variations circadiennes de performances cognitives (tâches d'addition, d'attention soutenue ou encore de mémoire à court terme) et la fluctuation systématique de mesures physiologiques, considérée comme marqueur de la rythmicité circadienne (notamment la température corporelle et l'expression endogène de l'hormone mélatonine chez l'homme). Cependant, des études ultérieures ont aussi montré que l'ampleur et la forme spécifique de la modulation dépendent, au moins partiellement, du domaine cognitif investigué (e.g., vigilance, arithmétique, temps de réaction sériel, rappel indicé, mémoire de travail, mémoire procédurale (voir [10] pour une revue), ainsi que des caractéristiques de la tâche utilisée (difficulté, longueur, feedback, etc.). De plus, un certain nombre de facteurs peuvent masquer ou affecter la variation journalière des performances cognitives, comme, par exemple, la motivation face à la tâche, le stress ou encore la consommation de boissons caféinées. II est à noter que, de la même manière que notre état de vigilance et nos performances cognitives, notre humeur varie aussi sur 24 heures, avec une humeur plus positive pendant la journée, atteignant globalement son maximum en fin de journée et son minimum en fin de la nuit [11].

D'autres études se sont intéressées aux fluctuations de performance en fonction du moment de l'évaluation et de la préférence matinale ou vespérale d'un individu (i.e., son chronotype ; voir section suivante pour une description détaillée de ce concept). Bien que cette approche n'offre pas un contrôle rigoureux sur l'influence des facteurs homéostatique et circadien, elle permet d'étudier les fluctuations journalières des performances dans les conditions de la vie quotidienne. De plus, ces études sont parvenues à mettre en évidence les domaines cognitifs particulièrement affectés par le moment de la journée. Ces travaux sont décrits plus en détail dans la section suivante.

\section{Différences interindividuelles dans notre horaire de veille-sommeil : impact sur la modulation journalière des performances cognitives}

Le chronotype d'une personne reflète principalement sa préférence dans les horaires de veille-sommeil. Le chronotype peut être évalué au niveau comportemental par des questionnaires permettant de distinguer de manière 
schématique les sujets «du matin » des sujets «du soir » $[12,13]$. Le profil chronotypique représente une importante source de variation interindividuelle dans notre profil de veille-sommeil : les extrêmes du matin se réveillent lorsque les extrêmes du soir s'endorment [13]. Ainsi le début et la fin de la nuit biologique varient fortement entre individus. Au-delà, les chronotypes se marquent aussi par des différences dans les périodes préférées de l'activité diurne, comme les heures de travail ou le moment de la journée pour effectuer un effort physique ou mental [14]. Bien sûr, l'origine de telles différences sur les préférences dans les horaires de veille-sommeil est multifactorielle. À côté d'une prédisposition génétique, les horaires de travail et les facteurs sociaux, mais aussi les conditions lumineuses peuvent contribuer au profil chronotypique d'un individu.

La phase du rythme circadien (voir encadré 1) des chronotypes extrêmes du matin et du soir sont avancés et retardés respectivement [15]. De plus, il semble que les chronotypes du matin présentent une période circadienne plus courte que les chronotypes du soir [16]. Un nombre croissant de données scientifiques suggère également que les chronotypes diffèrent dans la régulation homéostatique du sommeil. La dynamique du processus homéostatique de sommeil semble plus rapide chez les chronotypes du matin, par rapport aux chronotypes du soir. La conséquence en est une accumulation plus rapide de la pression de sommeil au cours de l'éveil chez les chronotypes du matin, menant à un niveau de fatigue plus important le soir et une diminution plus rapide de la pression homéostatique pendant le sommeil, favorisant potentiellement le réveil et l'activité aux heures matinales [17].

Le chronotype peut influencer nos comportements et notre fonctionnement cognitif au cours de la journée, c'est-à-dire que les capacités d'un individu s'exprimeront différemment selon le moment particulier de la journée auquel elles sont évaluées. La performance la meilleure (ou respectivement la plus mauvaise) pour un sujet coïncide avec un moment précis et déterminé de la journée, moment optimal (respectivement sous-optimal) variable en fonction de son chronotype (voir [10] pour une revue). Cette variabilité des performances a notamment été observée lors de tâches explorant divers aspects du fonctionnement exécutif [18], alors que la production de réponses routinières et surapprises ne semble pas ou moins affectée par la synchronisation entre le moment journalier préféré de la personne et le moment de l'évaluation. Finalement, il a été suggéré que les apprentissages se font de façon plus globale, englobant moins de détails et conservant plus d'informations superflues, à des moments sous-optimaux, par rapport aux moments optimaux de la journée [18].

L'âge est un facteur qui affecte les patterns d'activité circadienne. L'horloge biologique des adolescents présente une phase circadienne nettement retardée [19] par rapport aux jeunes enfants et aux personnes âgées. Par conséquent, le chronotype du soir est prédominant dans ce groupe d'âge. Sur le plan écologique, la préférence vespérale de la plupart des adolescents est en conflit avec les horaires scolaires imposés. Ce conflit peut mener à l'accumulation d'une dette de sommeil importante pendant les jours d'école [20]. De plus, les chronotypes du soir, et donc beaucoup d'adolescents, modifient le plus leurs horaires de veillesommeil entre les jours de travail ou d'école et les jours fériés. Ces modifications ont comme conséquence que I'adolescent adopte un rythme de veille-sommeil très irrégulier, pouvant mener à une privation de sommeil ou un niveau de fatigue important pendant la journée. L'impact de ces caractéristiques de veille-sommeil sur la performance cognitive chez les adolescents reste peu étudié à ce jour. Cependant, quelques études semblent indiquer que les adolescents du soir présentaient de meilleures performances à des tâches investiguant différents aspects du fonctionnement executif (e.g., Go-Nogo [tâche d'inhibition], lowa Gambling Task [tâche de prise de décision]) au cours de la soirée, par rapport à une évaluation réalisée le matin [21].

Alors que l'adolescence se marque par un décalage vers un chronotype du soir, le vieillissement est caractérisé par une avancée dans les horaires préférentiels de veille et de sommeil, un phénomène associé à une avance de la phase circadienne au niveau physiologique [22]. De plus, alors que les fonctions cognitives sensibles aux variations circadiennes tendent à s'améliorer au fil de la journée chez les sujets jeunes vespéraux, elles déclinent chez la majorité des personnes âgées. Les conséquences de cet effet de synchronisation entre le chronotype et le moment de l'évaluation cognitive sont apparemment plus importantes chez les personnes âgées que chez les sujets jeunes [18]. Dans cette optique, il a été observé, par exemple, qu'un certain nombre de différences de performance liées à l'âge, comme la reconnaissance en mémoire épisodique, sont fortement réduites, voire disparaissent, si l'évaluation se déroule tôt dans la matinée (i.e., moment optimal pour la plupart des personnes d'âge avancé et moment sousoptimal pour les sujets jeunes [18]).

Le vieillissement a également été associé à une réduction de l'amplitude des marqueurs circadiens (voir encadré 1), comme la mélatonine salivaire ou la température corporelle [22]. En parallèle, par rapport aux personnes plus jeunes, le niveau de vigilance, tel qu'investigué avec une tâche de temps de réaction, semble être moins affecté par une privation de sommeil au cours du vieillissement. Cette susceptibilité réduite aux effets néfastes du manque de sommeil a été observée surtout pendant la nuit biologique. En conséquence, dans des conditions de privation de sommeil, le ralentissement des temps de réponse classiquement observé lors du vieillissement est aboli [23].

En conclusion, bien que l'existence d'une modulation journalière des performances cognitives soit connue depuis longtemps, ce n'est que récemment que la prise en compte de l'existence de variations interindividuelles (telles que provoquées par le chronotype de la personne) sur cette modulation a suscité l'intérêt des chercheurs (voir la figure 2 pour une synthèse). De plus, un certain nombre d'études ont pu relever que ces différences dans les modulations journalières des performances cognitives en fonction du 


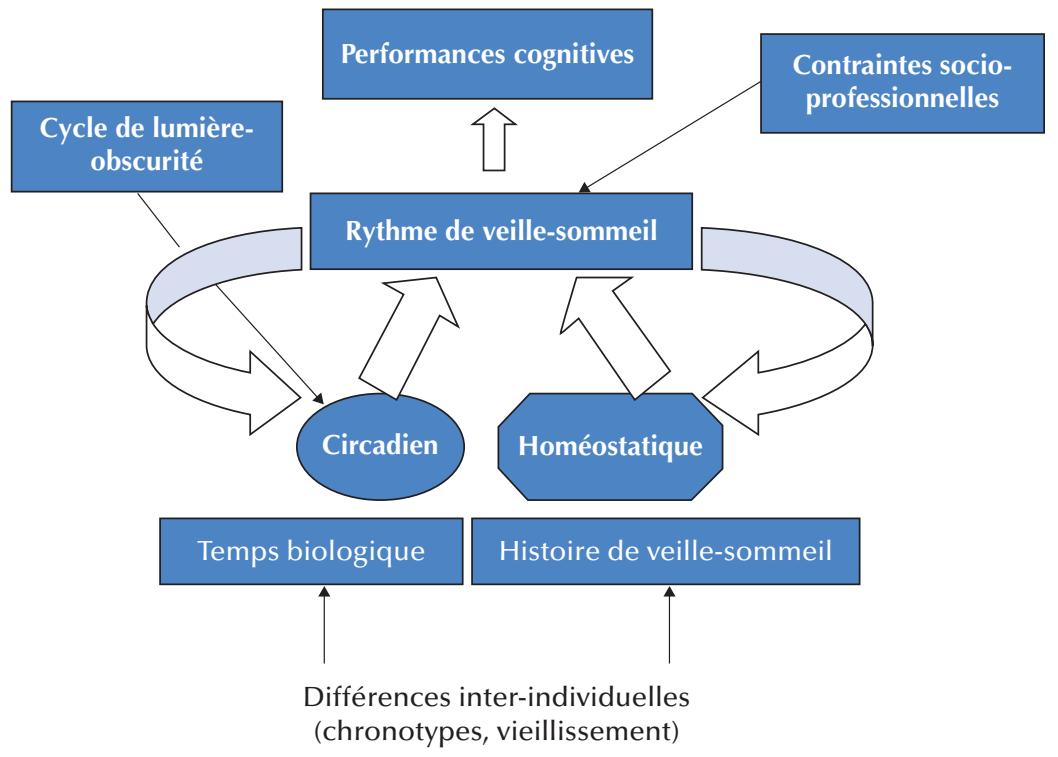

Figure 2. Régulation du cycle de veille-sommeil par le cycle de lumière-obscurité, les contraintes socioprofessionnelles, le processus circadien et homéostatique, son impact sur les performances cognitives et la modulation de cette régulation par le chronotype et l'âge (modifié de [37]).

chronotype sont aussi détectées au niveau du fonctionnement cérébral. Dans la section suivante, nous allons brièvement aborder ces études.

\section{Modulation journalière dans les corrélats cérébraux sous-jacents au fonctionnement cognitif}

Les premières évidences suggérant que notre état cérébral peut varier en fonction du moment de la journée viennent des études réalisées avec la technique de tomographie par émission de positrons (TEP) [24]. Il a, en effet, été observé que, comparé à celles du matin, les heures du soir étaient associées à un métabolisme accru au sein d'une série de régions impliquées dans la promotion de l'éveil (notamment l'hypothalamus et des régions du tronc cérébral). Des études plus récentes ont utilisé la technique de I'imagerie par résonance magnétique fonctionnelle (IRMf) dans le but d'explorer les corrélats cérébraux des modulations journalières sous-jacentes à une tâche cognitive. Dans cette perspective, une étude a détecté des modulations journalières de l'activité cérébrale dans une série de régions corticales, impliquées dans certains aspects spécifiques du contrôle attentionnel (e.g., régions fronto-insulaires, partie dorsale du cortex cingulaire antérieur, cortex préfrontal dorsolatéral) durant la réalisation d'une tâche de Stroop à cinq moments différents de la journée [25]. Le profil temporel d'activité cérébrale allait généralement dans le sens d'une réduction de l'activité du matin (6:00) jusqu'au soir (18:00), à l'exception de la dernière session (22:00), pour laquelle une augmentation était à nouveau détectée. Dans la même perspective, Peres et al. [26] ont observé que, pendant la réalisation d'une tâche motrice (finger tapping), une série de régions cérébrales motrices montrent des modulations en fonction du moment d'administration de la tâche (le matin, à midi, l'après-midi ou le soir). De plus cette modulation journalière dépendait du chronotype de la personne : plus la personne se caractérisait par un chronotype tardif, plus le moment de la journée caractérisé par un pic d'activité cérébrale survenait tardivement. Finalement, une étude réalisée par notre équipe a tenté de caractériser l'impact du moment de la journée et du chronotype sur les corrélats cérébraux sous-tendant une tâche d'attention soutenue et une tâche d'interférence cognitive [27, 28]. Étant donné que les chronotypes diffèrent dans leur régulation circadienne et homéostatique du cycle de veille-sommeil, une série de mesures supplémentaires (prélèvements de mélatonine, considérée comme marqueur de la phase circadienne, et enregistrement polysomnographique dans le but d'explorer la qualité, quantité et structure du sommeil précédant la session de testing) ont été réalisées en plus des acquisitions par IRMf. De plus, les horaires de testing ont été adaptés au chronotype spécifique de chaque personne, notamment pour éviter d'induire des différences du niveau de fatigue lié à un comportement de veille-sommeil non synchronisé avec les besoins physiologiques tels que dictés par l'horloge circadienne. En concordance avec la littérature, nous avons observé que les chronotypes extrêmes du matin présentent une dynamique plus rapide dans l'accumulation de la pression homéostatique de sommeil, ayant comme résultat un niveau de fatigue plus important, ainsi qu'une vigilance abaissée le soir, par rapport au chronotype extrême du 
soir. Ce phénomène était confirmé par les données issues de l'enregistrement polysomnographique (plus d'activité à ondes lentes en début de nuit chez les chronotypes du matin, indiquant une accumulation plus importante de la pression de sommeil). Au niveau cérébral, on a pu détecter que les chronotypes extrêmes du soir présentaient en soirée une activation plus importante de régions impliquées dans la promotion circadienne d'éveil (hypothalamus, locus coeruleus) durant la réalisation d'une tâche d'attention soutenue (tâche de temps de réaction simple d'une durée de dix minutes et un intervalle interstimulus randomisé entre deux et dix secondes). L'activation de ces régions pourrait donc expliquer le niveau d'alerte et de vigilance de base plus important des chronotypes du soir, même si les horaires de testing ont été adaptés aux profils chronotypique individuels.

Dans un deuxième temps, nous avons investigué l'effet du moment de la journée et du chronotype en utilisant une tâche d'interférence (le test de Stroop, version quatre couleurs avec items neutres, interférents et facilitateurs, voir [28] pour les détails). L'effet Stroop renvoie au fait que, si on demande à un sujet de nommer la couleur de l'encre dans laquelle est écrit un stimulus, il mettra significativement plus de temps pour dire « vert » en face du mot « ROUGE » écrit en vert qu'en face du stimulus contrôle « XXXX » écrit lui aussi en encre verte. Le sujet doit donc inhiber le mécanisme de lecture au profit de celui, moins automatique, de dénomination. Ce ralentissement produit par l'interférence de I'information sémantique activée automatiquement fut décrit initialement par Stroop (1935). De nouveau, une modulation journalière des régions cérébrales impliquées dans la tâche a été détectée (e.g., insula, gyrus cingulaire antérieur). Comme décrit plus haut, cette modulation était affectée par le chronotype de la personne, avec une diminution de l'activité du matin au soir chez les chronotypes extrêmes du matin et des profils d'activité stables ou légèrement augmentés du matin au soir chez les chronotypes extrêmes du soir.

En résumé, le moment de la journée, ainsi que le profil chronotypique de la personne affecte non seulement les performances, mais également les réseaux cérébraux sousjacents à la réalisation d'un effort cognitif. Afin d'illustrer I'intérêt au quotidien des études en chronobiologie, nous allons clôturer cette revue avec la présentation d'une série $\mathrm{d}^{\prime}$ applications cliniques.

\section{Un exemple de traitement issu de la chronobiologie : la luminothérapie}

Il est bien établi que la lumière exerce des effets importants sur le cerveau et notre bien-être [29]. La lumière n'est pas uniquement indispensable à la vision, mais joue aussi un rôle dans un ensemble de fonctions dites «non-visuelles » comme la synchronisation de notre horloge biologique avec I'alternance jour-nuit. La lumière constitue également un stimulant efficace pour l'éveil et elle est employée pour lutter contre la somnolence [30]. Les mécanismes qui sous-tendent ces effets bénéfiques de la lumière ne sont pas encore très connus. Un type de cellule sensible à la lumière (photorécepteur) appelé mélanopsine a été découvert dans I'œil [5]. Ce photorécepteur apparaît essentiel à la transmission de l'information lumineuse vers de nombreux centres du cerveau dits «non-visuels ». Les recherches en laboratoire ont montré que, sans ce photorécepteur, les fonctions non visuelles sont perturbées, et notamment I'horloge biologique est déréglée. La mélanopsine est particulièrement sensible à la lumière bleue et il a été montré que ce type de lumière s'avère le plus efficace en tant que stimulant de l'éveil. Des études en IRMf ont également montré une influence importante de la lumière sur les corrélats cérébraux sous-tendant un effort cognitif chez I'homme [29]. En effet, la lumière bleue est capable de moduler l'activité de structures sous-corticales impliquées dans la vigilance ainsi que dans toute une série de régions corticales, impliquées dans la réalisation de la tâche cognitive investiguée. Curieusement, même l'exposition à des écrans LED (contenant une quantité de lumière bleue plus importante que les écrans d'ordinateurs classiques) a des effets plus importants sur la vigilance subjective et la performance cognitive (e.g., tâche d'apprentissage de paires de mots) que les écrans classiques [31].

En parallèle à ces effets aigus de la lumière sur le comportement humain, l'exposition lumineuse a aussi le potentiel de décaler la phase de notre rythmicité circadienne. Le moment de l'exposition à la lumière est d'une importance cruciale dans ce contexte. De manière générale, la règle suivante peut être appliquée [32] : une phase circadienne plus tardive, et donc aussi une propension circadienne au sommeil plus tardive, peut être obtenue suite à une exposition lumineuse dans les heures du soir ou du début de la nuit. Un avancement de phase est obtenu suite à une exposition lumineuse dans les heures très matinales. De telle manière, la médecine du sommeil applique fréquemment de la luminothérapie pour le traitement de troubles du sommeil tels que le syndrome de retardement ou d'avancement de phase de sommeil (voir encadré 2 pour une définition de ces syndromes).

Un autre domaine d'application de la lumière concerne la prise en charge des troubles neurologiques associés à des perturbations majeures du rythme de veille-sommeil, comme par exemple dans la maladie d'Alzheimer (MA). En effet, la MA se définit notamment par une désorientation dans I'espace et dans le temps. Ainsi, une stabilisation de l'organisation temporelle du cycle de veille-sommeil par l'administration de lumière à des moments spécifiques de la journée peut s'avérer potentiellement bénéfique. En effet, les patients atteints de la MA souffrent souvent d'un sommeil nocturne fragmenté et l'activité diurne est perturbée par de multiples épisodes de sieste [33]. On a pu observer qu'une exposition lumineuse journalière sur plusieurs semaines (dans un home accueillant des personnes âgées atteintes de la MA) a le potentiel de stabiliser le profil 


\section{Encadré 2}

Le syndrome d'avance de phase du sommeil est un trouble du sommeil qui fait partie des troubles du rythme veille-sommeil. Dans ce trouble, les horaires du sommeil sont très avancés ou retardés par rapport aux horaires habituels et une somnolence apparaît en fin d'après-midi. La plainte est donc celle d'une insomnie matinale associée à une somnolence en seconde partie de journée. Le syndrome de retard de phase du sommeil représente le plus fréquent des troubles intrinsèques du rythme circadien. Sa fréquence est estimée à $0,5 \%$ de la population générale et à presque $10 \%$ des sujets se plaignant d'insomnie. La personne éprouve des grandes difficultés, voire une incapacité d'endormissement si le coucher a lieu à une heure dite normale. De même, une plainte est formulée pour se lever à une heure dite normale. On observe une somnolence diurne excessive durant la journée, des performances diurnes altérées, sauf en fin de journée. Cette plainte est liée à une dette de sommeil (durée de sommeil de moins de 5 heures si le sujet est soumis aux contraintes sociales). Les symptômes disparaissent si le sujet est libre de choisir ses horaires.

d'activité-repos de ces derniers, menant sur le plan cognitif à une réduction relative de l'avancement de la maladie tel qu'investigué par des scores à diverses échelles, ainsi que par une amélioration légère des symptômes dépressifs [34].

Finalement, la lumière, à travers son action sur le système circadien, présente un effet antidépresseur important $[35,36]$. Cette caractéristique a été exploitée de façon progressive depuis les années 1980. La luminothérapie est considérée comme un traitement de choix pour la dépression saisonnière. II est supposé que les effets activateurs et antidépressifs de la lumière sont principalement obtenus à travers des effets indirects sur la consolidation du cycle veille-sommeil. Cet outil est aussi de plus en plus utilisé pour le traitement des formes non saisonnières de dépression ou d'autres troubles psychiatriques, tels que les troubles de la personnalité (borderline), la dépression prénatale ou la boulimie nerveuse. Des informations plus détaillées sur la lumière en tant qu'outil thérapeutique peuvent être obtenues en consultant un site créé par des pionniers du domaine, dans le but de rendre cet outil accessible au grand public (www.cet.org).

\section{Conclusion générale}

Des périodes de veille et de sommeil survenant à des moments appropriés de notre cycle quotidien de 24 heures favorisent la santé, le bien-être ainsi que des performances cognitives adéquates (voir aussi figure 2 pour synthèse). II existe de plus en plus d'évidences montrant les effets délétères d'une désynchronisation entre rythmes circadiens et habitudes de vie, notamment parce que notre société nous impose fréquemment de travailler et de dormir à contretemps de nos besoins physiologiques. La prise en compte de telles modulations journalières sur le plan cognitif et cérébral, ainsi que de l'existence d'une importante variabilité interindividuelle, s'avère primordiale, notamment dans les circonstances (e.g., privation de sommeil, travail posté) ou maladies provoquant un déséquilibre entre les processus sous-jacents à la régulation du cycle de veille sommeil.

\section{Liens d'intérêts}

les auteurs déclarent ne pas avoir de lien d'intérêt en rapport avec cet article.

\section{Références}

1. Daan S, Beersma DGM, Borbély AA. Timing of human sleep: recovery process gated by a circadian pacemaker. Am J Physiol $1984 ; 246$ : R161-78

2. Van Dongen H, Vitellaro K, Dinges D. Individual differences in adult human sleep and wakefulness: leitmotif for a research agenda. Sleep $2005 ; 28: 479-96$.

3. Czeisler CA, Duffy JF, Shanahan TL, et al. Stability, precision, and near-24-hour period of the human circadian pacemaker. Science $1999 ; 284: 2177-81$

4. Duffy JF, Wright Jr KP. Entrainment of the human circadian system by light. J Biol Rhythms $2005 ; 20: 326-38$.

5. Hattar S, Liao HW, Takao M, et al. Melanopsin-containing retinal ganglion cells: architecture, projections, and intrinsic photosensitivity. Science $2002 ; 295$ : 1065-70.

6. Dijk DJ, Franken P. Interaction of sleep homeostatis and circadian rhythmicity: dependent or independent systems?. In : Kryger MHRT, Dement WC, éds. Principles and practice of sleep medicine. Philadelphia : Elsevier Saunders, 2005, 418-435.

7. Dawson D, Reid K. Fatigue, alcohol and performance impairment. Nature $1997 ; 17 ; 388: 235$.
8. Laird DA. Relative performance of college students is conditioned by time of day and day of week. J Exp Psychol 1925; 8:50-63.

9. Kleitman N, Jackson DP. Body temperature and performance under different routines. J Appl Physiol 1950; 3 :309-28.

10. Schmidt C, Collette F, Cajochen C, et al. A time to think: circadian rhythms in human cognition. Cogn Neuropsychol $2007 ; 24: 755-89$. 11. Boivin DB, Czeisler CA, Dijk DJ, et al. Complex interaction of the sleep-wake cycle and circadian phase modulates mood in healthy subjects. Arch Gen Psychiatry 1997; $54: 145-52$.

12. Horne JA, Östberg O. A self-assessment questionnaire to determine morningness-eveningness in human circadian rhythms. Int J Chronobiol $1976 ; 4$ : 97-110.

13. Roenneberg T, Wirz-Justice A, Merrow M. Life between clocks: daily temporal patterns of human chronotypes. I Biol Rhythms $2003 ; 18: 80-90$.

14. Taillard J, Philip P, Coste O, et al. The circadian and homeostatic modulation of sleep pressure during wakefulness differs between morning and evening chronotypes. I Sleep Res $2003 ; 12: 275-82$.

15. Kerkhof GA. Inter-individual differences in the human circadian system: a review. Biol Psychol $1985 ; 20: 83-112$. 


\section{Article de synthèse}

16. Duffy JF, Rimmer DW, Czeisler CA. Association of intrinsic circadian period with morningness-eveningness, usual wake time, and circadian phase. Behav Neurosci 2001; 115 : 895-9.

17. Mongrain V, Carrier J, Dumont M. Chronotype and sex effects on sleep architecture and quantitative sleep EEG in healthy young adults. Sleep $2005 ; 28: 819-27$.

18. Hasher L, Goldstein D, May C. It's about time: circadian rhythms, memory and aging. In : Ohta $\mathrm{Cl}$, éd. Human learning and memory: advances in theory and application. Kansas: Lawrence Erlbaum Associates, 2005, 199-217.

19. Carskadon MA. Sleep in adolescents: the perfect storm. Pediatr Clin North Am 2011; 58:637-47.

20. Wittmann M, Dinich J, Merrow M, et al. Social jetlag: misalignment of biological and social time. Chronobiol Int 2006; 23 : 497-509.

21. Hahn C, Cowell JM, Wiprzycka UJ, et al. Circadian rhythms in executive function during the transition to adolescence: the effect of synchrony between chronotype and time of day. Dev Sci $2012 ; 15: 408-16$

22. Munch M, Knoblauch V, Blatter K, et al. Age-related attenuation of the evening circadian arousal signal in humans. Neurobiol Aging $2005 ; 26: 1307-19$.

23. Adam M, Retey JV, Khatami R, et al. Age-related changes in the time course of vigilant attention during 40 hours without sleep in men. Sleep 2006; 29:55-7.

24. Buysse DJ, Nofzinger EA, Germain A, et al. Regional brain glucose metabolism during morning and evening wakefulness in humans: preliminary findings. Sleep $2004 ; 27: 1245-54$.

25. Marek T, Fafrowicz M, Golonka K, et al. Diurnal patterns of activity of the orienting and executive attention neuronal networks in subjects performing a Stroop-like task: a functional magnetic resonance imaging study. Chronobiol Int 2010;27:945-58.

26. Peres I, Vetter C, Blautzik J, et al. Chronotype predicts activity patterns in the neural underpinnings of the motor system during the day. Chronobiol Int $2011 ; 28: 883-9$.
27. Schmidt C, Collette F, Leclercq Y, et al. Homeostatic sleep pressure and responses to sustained attention in the suprachiasmatic area. Science $2009 ; 324$ : 516-9.

28. Schmidt $C$, Peigneux $P$, Leclercq $Y$, et al. Circadian preference modulates the neural substrate of conflict processing across the day. PLoS One 2012; 7 : e29658.

29. Vandewalle G, Maquet P, Dijk DJ. Light as a modulator of cognitive brain function. Trends Cogn Sci 2009; 13:429-38.

30. Cajochen C. Alerting effects of light. Sleep Med Rev $2007 ; 11: 453-64$

31. Cajochen C, Frey S, Anders D, et al. Evening exposure to a light-emitting diodes (LED)-backlit computer screen affects circadian physiology and cognitive performance. I Appl Physiol $2011 ; 110: 1432-8$.

32. Khalsa SB, Jewett ME, Cajochen $C$, et al. A phase response curve to single bright light pulses in human subjects. J Physiol 2003; 549: 945-52.

33. Van Someren EJ, Hagebeuk EE, Lijzenga C, et al. Circadian restactivity rhythm disturbances in Alzheimer's disease. Biol Psychiatry $1996 ; 40: 259-70$

34. Riemersma-van der Lek RF, Swaab DF, Twisk J, et al. Effect of bright light and melatonin on cognitive and noncognitive function in elderly residents of group care facilities: a randomized controlled trial. JAMA 2008;299:264255.

35. Terman M, Terman JS. Light therapy for seasonal and nonseasonal depression: efficacy, protocol, safety, and side effects. CNS Spectr $2005 ; 10: 647-63$.

36. Wirz-Justice A, Benedetti F, Berger $M$, et al. Chronotherapeutics (light and wake therapy) in affective disorders. Psychol Med $2005 ; 35: 939-44$

37. Dijk DJ, von Schantz M. Timing and consolidation of human sleep, wakefulness, and performance by a symphony of oscillators. I Biol Rhythms $2005 ; 20: 279-90$. 